\title{
Management of primary cardiac leiomyosarcoma in a young lady
}

\author{
Mehmet Onalan $^{1}$, Ahmet Demirkaya ${ }^{1}$, Kemal Behzatoglu ${ }^{1}$, and Ersin Erek ${ }^{1}$ \\ ${ }^{1}$ Acibadem University
}

May 11, 2020

\begin{abstract}
Cardiac leiomyosarcoma is an extremely rare tumor with a poor prognosis. We reported an 18- year-old girl with a left atrial leiomyosarcoma extending to right lower pulmonary veins. We performed complete tumor excision by the right anterolateral mini-thoracotomy approach using minimally invasive techniques. After pathological confirmation of the tumor, right lower lobectomy was performed with the same incision one week later to prevent a recurrence. Although no tumor remnant was found in the lobectomy specimen, adjuvant chemotherapy was started. No recurrence was detected during the 12-month follow-up.
\end{abstract}

\section{Introduction}

Cardiac tumors are a very rare condition with a frequency of $0.02 \%$. The prevalence ranges from $0.001 \%$ to $0.3 \%$, and only $25 \%$ is malignant (1). The most common histological types are angiosarcomas, followed by rhabdomyosarcomas, mesotheliomas, and fibrosarcomas.

Leiomyosarcoma appears for less than $1 \%$ of cases (1). Despite aggressive multidisciplinary treatment including surgery, chemotherapy, and radiotherapy, the prognosis is poor, with median survival about 12 months $(2,3,4)$. Metastasis is familiar, and up to $60 \%$ of cases have metastasis at the time of diagnosis (3). Adequate surgical resection, which may require lung resection ranging from lobectomy to pneumonectomy, is the mainstay of the treatment.

Clinical presentation is nonspecific; this makes early diagnosis difficult, and most cases are diagnosed on histology specimens and at autopsy. It is twice as common in females; the average age of occurrence is 45 years (range, 6 weeks to 77 years) (5).

In this case report, we present a successful management of leiomyosarcoma in a young lady.

\section{Case report}

An 18-year-old young lady with mild dyspnea and a 15-day history of treatment with a preliminary diagnosis of respiratory infection was admitted to our hospital. The diagnosis of pneumonia was suspected. In this context, a computerized tomographic (CT) scan revealed a 4 x $2,5 \mathrm{~cm}$ mass, involving the left atrium and the right inferior pulmonary vein, suggesting an intracardiac neoplasm. The patient was referred to us with a suspected diagnosis of a left atrial tumor. This was confirmed by a cardiac magnetic resonance imaging (MRI) that also showed a $4.4 \times 3 \times 2.2 \mathrm{~cm}$ mass in the left atrium extending to the right inferior pulmonary vein with gadolinium enhancement. A transthoracic and transesophageal echocardiogram showed a mass with little mobility in the left atrium extending to the right inferior pulmonary vein (Figure 1). The tumor was not obstructive, and both ventricular functions were preserved. We performed positron emission tomography (PET) CT to exclude malignancy and metastasis. PET CT revealed high fludeoxyglucose (FDG) uptake, and surgery was planned.

Because of the possible necessity for lung resection, the right submammarian thoracotomy approach was chosen. A double-lumen endotracheal tube was placed for unilateral ventilation during surgery. A right sub- 
mammary skin incision was performed to avoid damaging the mammary gland, after that subcutaneous fat and mammary gland were dissected from the fascia to expose the $4^{\text {th }}$ rib. After unilateral lung ventilation was initiated, the pericardium was opened vertically $1.5-2 \mathrm{~cm}$ anterior to the right phrenic nerve and suspended to ensure adequate exposure. After heparin was administered, aorta cannula was placed in the ascending aorta, and bicaval venous cannulas were inserted via the same thoracic incision. The cardioplegia needle was placed into the aortic root from the thoracic incision, and cardiopulmonary bypass (CPB) was started with mild hypothermia. A Chitwood clamp was used through the 4th intercostal space to clamp the ascending aorta, and cold blood cardioplegia was given. After the heart was arrested, the right atriotomy incision was performed, the interatrial septum was opened and the mass in the left atrium was reached. The tumor had a stable consistency, there was no necrosis or ulceration on its surface, and it was gently attached to the left atrial wall with a thin capsule. The tumor extended to the bifurcation of the right lower pulmonary vein branches, and it could be excised entirely with its stalk (Figure 2). Cold saline was used to rinse the heart chambers, and careful examination was performed to ensure complete resection. After the de-airing procedure, the interatrial septum and the right atriotomy was closed primarily. The aortic cross-clamp was removed, and the heart was reperfused.

The tumor was evaluated with the frozen section examination but couldn't be classified as benign or malignant neoplasm during the operation. Therefore, we didn't perform lymph node dissection and right lower lobectomy. Cessation of CPB was uneventful, and the thoracotomy was closed. The patient recovered smoothly, she was transferred to ward on postoperative day 1, and discharged from the hospital on postoperative day 6. On the histopathologic evaluation, multiple sections revealed pleomorphic spindle tumor cell fascicles with nuclear hyperchromasia. Atypical mitosis was seen (14 mitotic activity/10 HPF) with coagulation necrosis. Actin and Desmin were positive for reaction, but CD34 and S100 were negative (Figure 3 ). A total of $30 \%$ positivity was shown by tumor cells for Ki-67, and these morphological features were consistent with a diagnosis of leiomyosarcoma.

After histopathologic confirmation of leiomyosarcoma, we planned right lower lobectomy to be sure complete resection of the tumor and to prevent a recurrence. The same incision was used. Right lower lobectomy with right inferior pulmonary vein excision, mediastinal lymph node dissection and partial left atrial resection was performed without CPB. The samples of tissues were taken from the surgical margin. The thoracotomy was closed, and the patient was transferred to the cardiovascular intensive care unit. The postoperative period was uneventful, and the chest tube was removed on postoperative day 5 . The patient discharged from the hospital on a postoperative day 7 . Histopathological examination revealed that the resected pulmonary vein and left atrial specimen borders were histologically clear.

Thorax and abdomen MR scans revealed no metastases in the postoperative early period. According to a multidisciplinary staff, adjuvant chemotherapy consisting of four cycles of Doxorubicin and Ifosfamide was prescribed by the medical oncology department. Moreover, she had adjuvant cardiac radiotherapy (54 Gy in 27 fractions) at postoperative $5^{\text {th }}$ month. The patient is regularly followed, no metastasis was detected in PET CT and thoracic MR scans, after 4 and 6 months follow up, respectively. After a follow-up of 12 months, she still has no clinical or radiological evidence of recurrence.

\section{Discussion}

We present a rare case of cardiac leiomyosarcoma in a young lady who treated surgically, followed by adjuvant chemotherapy. The patient is still alive without metastasis after 9 months of follow-up.

There are few reports of limited cases in the literature about primary cardiac leiomyosarcoma. Wang et al. reviewed all the literature and identified 79 instances derived from 72 unique reports with primary cardiac leiomyosarcoma (4). In this study, the median age at diagnosis was 48 with a female predominance, and the symptom of obstruction was the most common complaint at diagnosis. The recurrence-free survival and the 5-year overall survival rates were $14.7 \%$ and $25.4 \%$, respectively. They showed that overall survival was affected by age, surgery, and adjunct chemotherapy or radiotherapy in this study. In our case, the patient had mild dyspnea and a history of treatment with a respiratory infection. The diagnosis was suspected on 
the findings of a CT performed for pneumonia.

Echocardiography is usually the initial imaging modality for cardiac masses. It may show its extent, location, and hemodynamic effects (Figure 1). CT scan and cardiac MRI supply additional instructions about morphology, location, and extent of the mass (Figure 2) (6). CT scan is helpful to evaluate extracardiac extent or metastasis, and cardiac MRI has been shown to differentiate the tumor from a thrombus by contrast enhancement (3). Moreover, MRI is more useful to assess myocardial involvement. For the differential diagnosis of a mass lesion, PET CT helps differentiate the results of CT and MRI (7). In our case, we used PET CT and MRI for the definitive diagnosis of the cardiac tumor and for the follow-up.

The most common cardiac chamber of cardiac leiomyosarcomas in the left atrium (51\%), like our case. A biopsy is the gold standard for histological confirmation, but this step can sometimes be overcome, especially in cardiac masses, and then diagnosed by histopathological examination of the resected mass (6).

The best treatment strategy is not certain; but, early diagnosis and adequate surgical resection remain the mainstay for the treatment of this tumor in the literature (3). Adjuvant radiotherapy and chemotherapy have been shown to prolong survival and improve disease control in some cases (7). Frozen section guided excision border of $1 \mathrm{~cm}$ for all left atrial tumors was suggested by Marin and colleagues for better survival (7). Adequate excision of a pulmonary vein tumor may require lung resection ranging from lobectomy to pneumonectomy (3). In our case, the left atrial tumor was extending to the branches of the pulmonary veins, so the right inferior lobectomy with right inferior pulmonary vein excision was performed to reach the clean surgical margin. Adjuvant radiotherapy and chemotherapy was given to our patient by multidisciplinary staff decision.

The conventional median sternotomy approach is widely used in cardiac tumors operations. This approach has great exposure; on the other hand, it also coexists with some troubles, such as poor cosmetic effect, sternal dehiscence and risk of postoperative mediastinitis (8). Since Ko et al. reported the cardiac myxoma excision via the right anterolateral mini-thoracotomy approach two decades ago (9), minimally invasive approaches have been progressively performed to the treatment of cardiac tumors. However, because of the rareness of primary cardiac tumors, there are only limited reports in the literature. In addition, there are some concerns about incomplete tumor resection with minimally invasive surgery due to insufficient minimally invasive experience, and the clinical benefit of this approach is still controversial, especially for cardiac masses. Luo et al. conducted a study on 50 patients with primary benign cardiac tumors who underwent either conventional or minimally invasive surgery from 2008 to 2018 (10). In this study, there was no significant difference in aortic cross-clamp time and cardiopulmonary bypass time between the two groups and the duration of postoperative intubation time, intensive care unit stay, and hospital stay were shorter in the minimally invasive group, but the differences were not statistically significant.

The necessity of lung resection is high for this type of tumor, and it may difficult to perform from median sternotomy. Additional right thoracotomy may be needed. We suggest that the right submammarian thoracotomy approach is feasible and effective for both cardiac tumor resection and right lower lobectomy.

The recurrence rate is high in cardiac leiomyosarcomas despite optimal surgical resection of the primary tumor. According to the literature of cardiac leiomyosarcomas, the mean survival time of patients who underwent surgery and chemotherapy was about 12 months $(2,3,4)$.

In our case above, she is still alive, and there is no evidence of local or distant recurrence or metastasis after a follow-up of 12 months.

\section{Conclusion}

Cardiac leiomyosarcoma is an extremely rare tumor with a poor prognosis, and frozen section guided complete resection is the key to the treatment. Adjuvant chemoradiotherapy may further prolong survival and regular echocardiography should be followed up after the operation. The right submammarian thoracotomy approach has the advantages of its less invasive nature and suitability for complete tumor resection with lobectomy. 


\section{References}

1. Patel J, Sheppard MN. Pathological study of primary cardiac and pericardial tumors in a specialist UK Centre: surgical and autopsy series. Cardiovasc Pathol. 2010;19(6):343-52.

2. Lv Y, Pang X, Zhang Q, Jia D. Cardial leiomyosarcoma with multiple lesions

involved: a case report. Int J Clin Exp Pathol. 2015;8(11):15412-6.

3. Gukop P, Frassetto G, Karapanagiotidis G, et al.: Leiomyosarcoma of pulmonary vein presenting as left atrial mass: An outline of management principles. Case Rep Cardiol 2012:396319, 2012.

4. Wang J-G, Cui L, Jiang T, Li Y-J, Wei Z-M. Primary cardiac leiomyosarcoma: an analysis of clinical characteristics and outcome patterns. Asian Cardiovasc Thorac Ann. 2015;23(5): 623-30.

5.Y. Hattori, T. Iriyama, K. Watanabe, K. Negi, I. Takeda, and S. Sugimura, "Primary cardiac sarcoma - two case reports," Japanese Circulation Journal, 2000; 64(3) 222-224.

6. Behi K, Ayadi M, Mezni E, Meddeb K, Mokrani A, Yahyaoui Y, et al. Two years survival of primary cardiac leiomyosarcoma managed by surgical and adjuvant therapy. Clin Sarcoma Res. 2017 7:5.

7. Morin JE, Rahal DP, Huttner I. Myxoid leiomyosarcoma of the left atrium: a rare malignancy of the heart and its comparison with atrial myxoma. Canadian Journal of Cardiology. 2001;17(3): 331-6.

8. Lee HP, Cho WC, Kim JB, Jung SH, Choo SJ, Chung CH, et al. Surgical outcomes of cardiac Myxoma: right Minithoracotomy approach versus median sternotomy approach. The Korean journal of thoracic and cardiovascular surgery. 2016;49(5):356-60.

9. Ko PJ, Chang CH, Lin PJ, Chu JJ, Tsai FC, Hsueh C, et al. Video-assisted minimal access in excision of left atrial myxoma. Ann Thorac Surg. 1998;66(4):1301-5.

10. Luo C, Zhu J, Bao C, Ding F, Mei J. Minimally invasive and conventional surgical treatment of primary benign cardiac tumors. Journal of Cardiothoracic Surgery. 2019;14:76.

\section{Figure Legends}

1. Transesophageal echocardiography is showing a hyperechoic mass lesion, measuring $2,8 \times 2.6 \mathrm{~cm}$, in the left atrium (A). Cardiac MRI is showing the left atrial mass extending into the pulmonary vein (B, C).

2. The view of cardiac tumor in the left atrium after interatrial septal incision (A). The complete excision of the tumor via the right anterolateral mini-thoracotomy approach (B). Resected left atrial tumor showed a smooth white surface without any necrosis or ulceration $(\mathrm{C})$.

3. Hematoxylin \& eosin stains showed a sarcomatous lesion with prominent inflammatory reaction and epithelioid neoplastic cells (orig. x400) (A). Immunohistochemical stains showed strong and diffuse cytoplasmic immunoreactivity with smooth-muscle actin (orig. x100) (B).

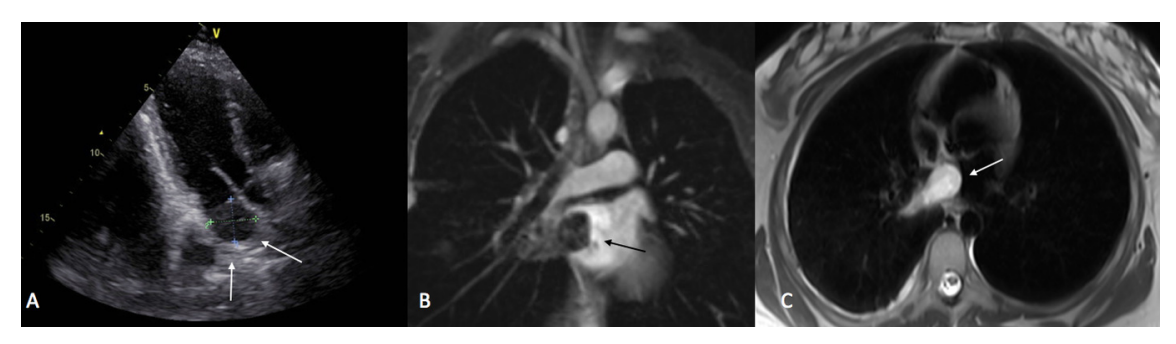



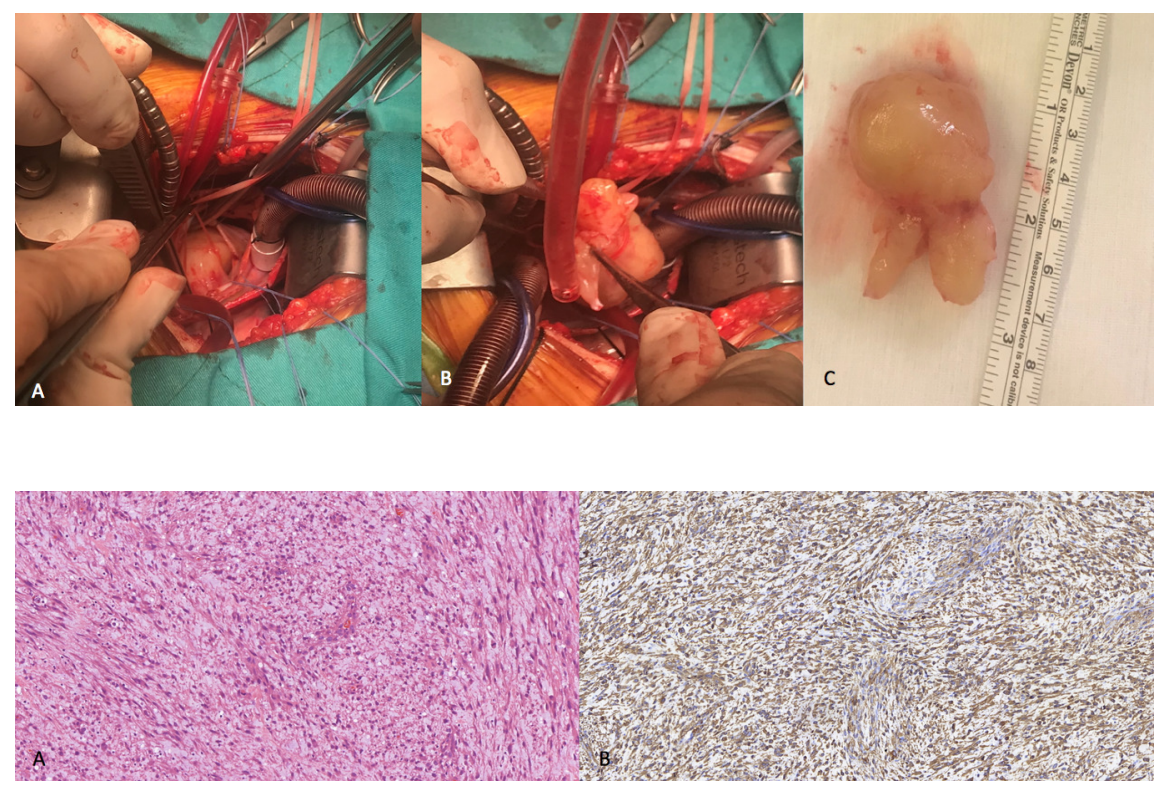